Check for updates

Cite this: RSC Adv., 2019, 9, 35990

\title{
Modulating interfacial charge distribution and compatibility boosts high energy density and discharge efficiency of polymer nanocomposites $\uparrow$
}

\author{
Tao Zhang, Mengfan Guo, Jianyong Jiang, Xueyou Zhang, Yuanhua Lin, \\ Ce-Wen Nan (iD) and Yang Shen (iD*
}

Polymer nanocomposite dielectrics, composed of polymer matrices with high breakdown strength and nanofillers with high dielectric constant, can achieve outstanding energy density. However, the great difference of intrinsic surface properties between the polymer and nanofillers will lead to poor compatibility and thus damage the dielectric properties of the composites. Introducing a transition layer to the filler surface can effectively reduce the degree of mismatch. In this work, we use a "direct in situ polymerization" method to synthesize core-shell $\mathrm{BaTiO}_{3}$ nanoparticles (BTO_nps) with three types of stable and dense fluoro-polymer shells, e.g., poly(2,2,2-trifluoroethyl methacrylate) (PTFEMA), poly(2,2,3,4,4,4-hexafluorobutyl methacrylate) (PHFBMA), and poly( $1 H, 1 H, 7 H$-dodecafluoroheptyl methacrylate) (PDFHMA), and individually disperse them into the poly(vinylidene fluoride-co-hexafluoro propylene) (P(VDF-HFP)) matrix. Benefitting from the good interaction between the fluorine-containing segments in the shell polymer and the matrix segments, the dispersion of core-shell BTO_nps and their compatibility with P(VDF-HFP) are improved, which leads to a significant improvement in the dielectric properties of the nanocomposites. The results show that BTO@PDFHMA/P(VDF-HFP) composite exhibits an ultrahigh energy density of $16.8 \mathrm{~J} \mathrm{~cm}^{-3}$ at $609 \mathrm{MV} \mathrm{m}^{-1}$ with particle loading amount of $15 \mathrm{wt} \%$, compared to $11.5 \mathrm{~J} \mathrm{~cm}^{-3}$ at $492 \mathrm{MV} \mathrm{m}^{-1}$ for a conventional solution blended BTO/P(VDF-HFP) composite. Meanwhile, the discharge efficiency is enhanced from $\sim 62$ to $\sim 78 \%$. It is elucidated that the core-shell strategy can achieve improved particle dispersion and dielectric properties. We consider that this simple method can well achieve the preparation of core-shell structures in dielectric nanocomposites.

Received 1st September 2019 Accepted 25th October 2019

DOI: 10.1039/c9ra06933j

rsc.li/rsc-advances
It can be seen from the formula (1), that the applied electric field $(E)$ and the electric displacement $(D)$ of the dielectrics determine the maximum $U_{\mathrm{e}}$ value. For a dielectric material, $D$ has a relationship with the dielectric constant $\left(\varepsilon_{\mathrm{r}}\right)$ as $D=\varepsilon_{0} \varepsilon_{\mathrm{r}} E$, where $\varepsilon_{0}$ and $\varepsilon_{\mathrm{r}}$ are the vacuum permittivity and the effective permittivity of the dielectrics, respectively. ${ }^{10}$ Thus, it is very important to improve the dielectric constant of polymer dielectric materials for high energy storage density. However, the low intrinsic dielectric constants $\left(\varepsilon_{\mathrm{r}}<3\right)$ of polymers are non-negligible, even though they have high breakdown strength. For instance, the $U_{\mathrm{e}}$ of commercial biaxially oriented polypropylene (BOPP) is only $\sim 2 \mathrm{~J} \mathrm{~cm}^{-3}$ even at a high $E_{\mathrm{b}}$ of 600 $\mathrm{MV} \mathrm{m}^{-1}$, and low $U_{\mathrm{e}}$ limits its application in energy storage devices. ${ }^{11}$

Recently, polar polymers such as poly(vinylidene fluoride) (PVDF)-based ferroelectric polymers are considered as a promising dielectric energy storage material, due to their relatively high dielectric constants compared with non-polar polymer. ${ }^{\mathbf{1 2 , 1 3}}$ But their dielectric constants are still relatively low. In order to achieve higher $\varepsilon_{\mathrm{r}}$ of PVDF-based polymers, an effective method is to fabricate nanocomposites by introducing nano-sized inorganic fillers with high dielectric constants into polymer 
matrices. ${ }^{\mathbf{1 4 - 1 6}}$ However, in the actual process, direct mixing of inorganic nanoparticles with the polymer matrix will bring a few problems. For example, the large contrast in their dielectric properties, rigidities and chemical bonding types will cause the agglomeration of nanoparticles, and the particle agglomerations will induce inhomogeneity of the local electric field distribution which causes early breakdown of the nanocomposite at an electric field well below the intrinsic $E_{\mathrm{b}}$ of the polymer matrix. ${ }^{17}$ Moreover, the uneven dispersion of nanofillers is usually accompanied by structural imperfections, voids and impurity ions, etc. ${ }^{17}$ To solve these issues, tremendous efforts have been made to improve the compatibility between the nanoparticles and the polymer matrix, among which surface modification is one of the most promising approaches. ${ }^{\mathbf{1 8}}$

Generally, the modifier for surface modifications will contain some functional groups, such as $-\mathrm{OH},-\mathrm{NH}_{2},-\mathrm{COOH}$, etc., and then the modifier will be bound to the surface of the filler by hydrogen bonding or electrostatic interaction. ${ }^{19}$ Finally, the interaction between particles and polymer matrix is improved by introducing modifier. So far, the modifiers commonly used in the surface modification process are hydrogen peroxide $\left(\mathrm{H}_{2} \mathrm{O}_{2}\right){ }^{20}$ Phosphonic acids, ${ }^{21,22}$ silane coupling agents, ${ }^{23}$ etc. Unfortunately, although these modifiers lead to an improvement in the compatibility between the matrix and the nanoparticles, they will also bring high leakage current and thus high dielectric loss. ${ }^{24}$ Therefore, some researchers adjust the functional groups in the molecular chain of modifier to improve its compatibility with the polymer matrix, thereby improving the related dielectric properties of the nanocomposite. Wang et al. studied the effects of various carboxylic acid modifiers on the properties of nanocomposites. It was found that the type, number, and position of functional groups in the modifier molecules resulted in significantly different modification influences. ${ }^{\mathbf{1 9 , 2 5}}$ Dogan et al. used organophosphorus with electron-rich and electron-poor groups to investigate how the structure of the interfacial layer influences the dielectric properties of the composite. The results show that the modification of the phosphoric acid ligand containing positively charged ligands will cause the charge transfer to each other at the interface, which can effectively reduce the leakage current and dielectric loss and increase the energy storage density of the composite material. ${ }^{26}$ However, the binding force between the particles and the matrix is still insufficiently strong, due to the small size of the modifier itself.

Consequently, inorganic nanoparticles with a layer of polymer shell are employed to facilitate better combination between fillers and matrices. ${ }^{27-30}$ Huang and Jiang have used in situ atom transfer radical polymerization (ATRP) and reversible additionfragmentation chain transfer (RAFT) to prepare a series of uniformly dispersed core-shell inorganic nanoparticles, such as $\mathrm{BaTiO}_{3} @ \mathrm{PMMA} \mathrm{BaTiO}_{3} @ \mathrm{PS}$ and BaTiO 3 @HBP@PMMA..7,31,32 Benefited from the polymer shell, the corresponding composites all achieved high dielectric constant and low dielectric loss. However, although the shell structure prepared by the in situ polymerization method is stable and reliable, the disadvantage is that the preparation process is complicated.
Recently, Zhang et al. designed and synthesized a fluoricliquid-crystalline polymer to tailor the surface of filler, and a high energy discharge density of nanocomposite was obtained due to the modification of the PTFMPCS layer thickness. ${ }^{33}$ It demonstrated that introducing fluorine-containing shell as the transition layer is an effective strategy to improve the compatibility between fluorine-containing matrix and nanofiller and thus obtain a high-performance nanocomposite. In this article, three kinds of BTO_nps core-shell structures with different fluoro-polymer shells are prepared by "direct in situ polymerization" method. The fluoro-polymer shells are poly(2,2,2trifluoroethyl methacrylate) (PTFEMA), poly $(2,2,3,4,4,4$ hexafluorobutyl methacrylate) (PHFBMA), poly $(1 \mathrm{H}, 1 \mathrm{H}, 7 \mathrm{H}$ dodecafluoroheptyl methacrylate) (PDFHMA), respectively. By "in situ” introducing BTO_nps during the polymerization of the monomer, a dense and stable shells consisting of different fluoro-polymers can be successfully introduced on the surface of the BTO_nps. More importantly, compared with other coated polymers, these three fluoro-polymers all have fluorinecontaining branched chains, which makes their segment structure similar to poly(vinylidene fluoride-co-hexafluoro propylene) (P(VDF-HFP)). Thus, the fluoro-polymer shells can interact well with $\mathrm{P}(\mathrm{VDF}-\mathrm{HFP})$ matrix, and provide a strong adhesive force between particle and matrix. Hence, not only the uniform dispersion of BTO_nps, the tight bonding between particles and matrix is also achieved. As a result, compared with BTO/P(VDF-HFP) nanocomposite without core-shell structure (energy density of $11.5 \mathrm{~J} \mathrm{~cm}^{-3}$ at $492 \mathrm{MV} \mathrm{m}^{-1}$ ), the dielectric constant and the breakdown strength are significantly improved and an ultrahigh energy density of $16.8 \mathrm{~J} \mathrm{~cm}^{-3}$ is achieved at $609 \mathrm{MV} \mathrm{m}^{-1}$ for BTO@PDFHMA/P(VDF-HFP).

\section{Experimental section}

\section{Materials}

BTO_nps (about $50 \mathrm{~nm}$ in diameter) were supplied by the Shandong Sinocera Functional Material Co., Ltd. 2,2,2-Trifluoroethyl methacrylate (TFEMA), 2,2,3,4,4,4-hexafluorobutyl methacrylate (HFBMA) and $1 H, 1 H, 7 H$-dodecafluoroheptyl methacrylate (DFHMA) were purchased from Harbin Xuejia fluorin silicon Chemical Co., Ltd. Unless otherwise specified, all the chemicals were purchased from China National Chemicals Corporation Ltd.

\section{Preparation of core-shell structured BTO nanoparticles}

Three kinds of BTO_nps with core-shell structures were synthesized by chemical polymerization. In a typical procedure, the mixture of $2 \mathrm{~g}$ BTO_nps and $80 \mathrm{~mL}$ aqueous solution of $\mathrm{H}_{2} \mathrm{O}_{2}$ (30 wt $\%$ ) was added into a $25 \mathrm{~mL}$ round bottom flask. After stirring at $70{ }^{\circ} \mathrm{C}$ for $8 \mathrm{~h}$, the mixture was centrifuged at $10000 \mathrm{rpm}$ for $10 \mathrm{~min}$ and then was washed three times with deionized water. Finally, the BTO nanoparticles with hydroxylation (BTO-OH) were dried under vacuum at $80{ }^{\circ} \mathrm{C}$ for $12 \mathrm{~h}$.

$20 \mathrm{~g}$ of pre-synthesized BTO-OH nanoparticles were added into $100 \mathrm{~mL} \mathrm{~N}, \mathrm{~N}$-dimethylformamide (DMF) with ultrasonic treatment for $30 \mathrm{~min}$ for homogeneous mixing. Then $0.35 \mathrm{~g}$ of azobisisobutylonitrile (AIBN) initiator was added with magnetic 
stirring for $10 \mathrm{~min}$, followed by adding $60 \mathrm{~g}$ of TFEMA monomers. The mixture solution was kept stirring under $\mathrm{N}_{2}$ atmosphere at $70{ }^{\circ} \mathrm{C}$ for $4 \mathrm{~h}$. Subsequently, the flask was placed under the ice water to prevent polymerization. The BTO@PTFEMA nanoparticles were obtained by centrifugation at $10000 \mathrm{rpm}$ for $10 \mathrm{~min}$ and washed with DMF three times. The products were dried under vacuum at $80{ }^{\circ} \mathrm{C}$ for $12 \mathrm{~h}$. The similar procedure was applied to synthesize BTO@PHFBMA and BTO@PDFHMA nanoparticles with different monomers.

\section{Fabrication of P(VDF-HFP) based nanocomposite films}

The polymer nanocomposite films with three different kinds of core-shell structured nanofillers were fabricated by the nonequilibrium process. $^{34}$ Specifically, take fabrication of BTO@PTFEMA/P(VDF-HFP) for example, the P(VDF-HFP) powders (Arkema, France, Kynar Flex 2801 with 10 wt\% HFP) were firstly thoroughly dissolved in the mixed solvent of $\mathrm{N}, \mathrm{N}$ dimethylmethanamide (DMF) and acetone. With the aid of ultrasonic treatment, BTO@PTFEMA nanoparticles were then dispersed homogenously in the P(VDF-HFP) solution. After stirring for $12 \mathrm{~h}$, the obtained solution was transferred into a syringe as electrospinning precursor solution. Then the electrospinning process was carried out under an applied field of $1.0 \mathrm{kV} \mathrm{cm}{ }^{-1}$ with flow rate of $1.0 \mathrm{~mL} \mathrm{~h}^{-1}$ for $2 \mathrm{~h}$. The collected composite fibers were hot-pressed at $200{ }^{\circ} \mathrm{C}$ under the pressure of $10 \mathrm{MPa}$ for $1 \mathrm{~h}$. The as-pressed composite films were annealed at $220^{\circ} \mathrm{C}$ for $5 \mathrm{~min}$ followed by a quenching process in ice-water. The similar process was applied to fabricate the BTO@PHFBMA/P(VDF-HFP) and BTO@PDFHMA/P(VDF-HFP) nanocomposite films. The thickness of the final nanocomposite films was $\sim 12 \mu \mathrm{m}$.

\section{Characterization}

The structures of the BTO nanoparticles (BTO@PTFEMA, BTO@PHFBMA and BTO@PDFHMA) were characterized by transmission electron microscopy (TEM, JEM2100F). The contents of the shell polymer in the core-shell nanoparticles were determined by thermal gravimetric analysis (TGA, TGA/ DSC, Metter-Toledo, Switzerland) within the temperature range from $30{ }^{\circ} \mathrm{C}$ to $700{ }^{\circ} \mathrm{C}$. The morphology of nanofibers, films and the thickness of the films were characterized with scanning electron microscopy (SEM, Zeiss, MERLIN VP Compact). The chemical structure of shell polymer coated on the surface of BTO nanoparticles was characterized by Fourier-transform infrared spectroscopy (FTIR, VERTEX 70V, Brucker).

\section{Measurements of dielectric properties}

The dielectric permittivity and loss of the P(VDF-HFP)-based nanocomposites were measured by a HP 4294 A impedance analyzer (Agilent Technologies, Inc.) at room temperature with the measurement frequency range from $10^{2} \mathrm{~Hz}$ to $10^{7} \mathrm{~Hz}$. The electric displacement-electric field $(D-E)$ loops were measured at $10 \mathrm{~Hz}$ with a Premier II ferroelectric test system (Radiant Technologies, Inc.). The leakage current and the electric breakdown strength measurements were performed on the same ferroelectric test system. The temperature dependent and frequency dependent of the imaginary electric modulus spectra $\left(M^{\prime \prime}\right)$ of the films were measured by a Novocontrol (Keysight

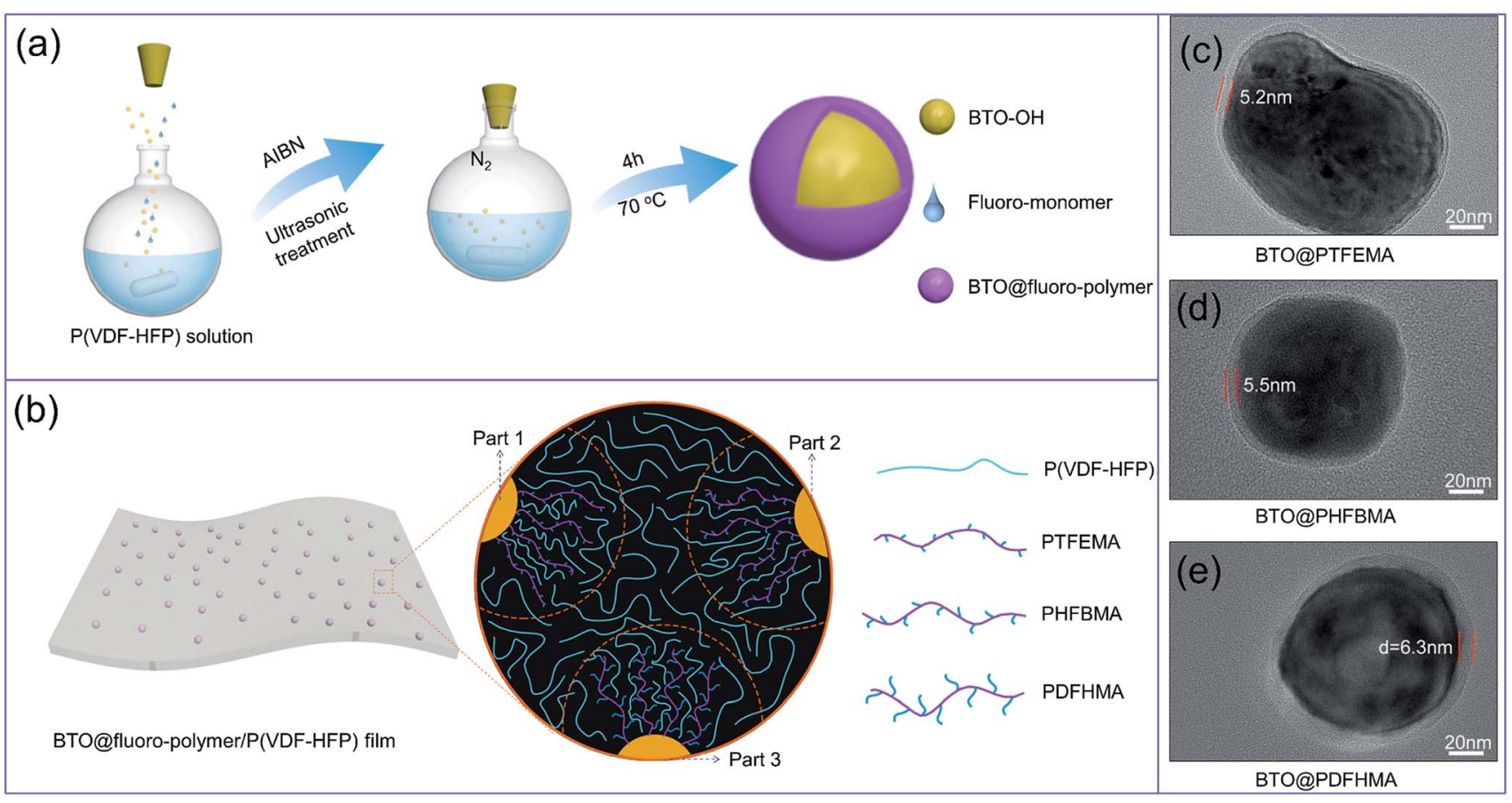

Fig. 1 Schematic illustration of (a) the fabrication process of BTO@fluoro-polymer nanoparticles and (b) the interaction between the shell segment structure and P(VDF-HFP) chain segment. TEM images of (c) BTO@PTFEMA, (d) BTO@PHFBMA and (e) BTO@PDFHMA nanoparticles, respectively. 
Technologies, Inc.) with the frequency range from $10^{-2} \mathrm{~Hz}$ to $10^{7} \mathrm{~Hz}$ and the temperature range from $-30^{\circ} \mathrm{C}$ to $130{ }^{\circ} \mathrm{C}\left(20^{\circ} \mathrm{C}\right.$ is the interval).

\section{Result and discussion}

The BTO@fluoroalkyl methacrylate core-shell structured nanoparticles were fabricated by a simple chemical polymerization reaction. The preparation process is shown in Fig. 1a. Here, three kinds of fluoroalkyl methacrylate monomers (TFEMA, HFBMA and DFHMA) are employed to investigate the effect of the side chain length of monomer on dielectric properties of polymer nanocomposites. Fig. 1 b shows a schematic representation of the interaction between the shell segment structure and $\mathrm{P}(\mathrm{VDF}-\mathrm{HFP})$ chain segment of three fluorinecontaining core-shell particles in the nanocomposite films (part 1, part 2, and part 3 of the dotted circle respectively represent the state of the three particles in the P(VDF-HFP) matrix). As we can see from Fig. 4c, the shell polymers formed by three monomers all have fluorine-containing branched chains, which are highly similar to P(VDF-HFP) chain segments. Therefore, the fluoro-polymer shells can interact well with $\mathrm{P}(\mathrm{VDF}-\mathrm{HFP})$, and the interaction between the shell and the matrix is more intense as the length of the fluorine-containing segment increases. The TEM images of as-fabricated core-shell nanoparticles are exhibited in Fig. 1c-e. As shown, a stable and dense layer of polymer shell is tightly coated on the BTO_nps surface in all three cases. By controlling the concentration of monomers, the thicknesses of shell layer on the surface of BTO_nps are controlled within 5 to $6 \mathrm{~nm}$. Therefore, the TEM morphology of the nanoparticles directly proves that the preparation of the core-shell structure is successful.

In order to understand the chemical structure on the surface of the nanoparticles, the chemical structure of fluoro-polymer shells is characterized by the FTIR (Fig. 2a). It is found that compared with BTO_nps with bare surface, nanoparticles with core-shell structure exhibit significant absorption signals between 1000 and $2000 \mathrm{~cm}^{-1}$ in FTIR spectra. Firstly, all coreshell nanoparticles possess a new absorption bands located at $1750 \mathrm{~cm}^{-1}$, which is associated with $\mathrm{C}=\mathrm{O}$. Besides, each coreshell nanoparticles have their own characteristic adsorption peaks, namely $1186 \mathrm{~cm}^{-1}$ for the $-\mathrm{CF}_{3}$ of PTFEMA, $1190 \mathrm{~cm}^{-1}$ for the $-\mathrm{CF}_{2}$ of PHFBMA and $1300 \mathrm{~cm}^{-1}$ for the $-\mathrm{CF}_{3}$ of PDFHMA. ${ }^{35-37}$ Thus, the results indicate that the fluoroalkyl
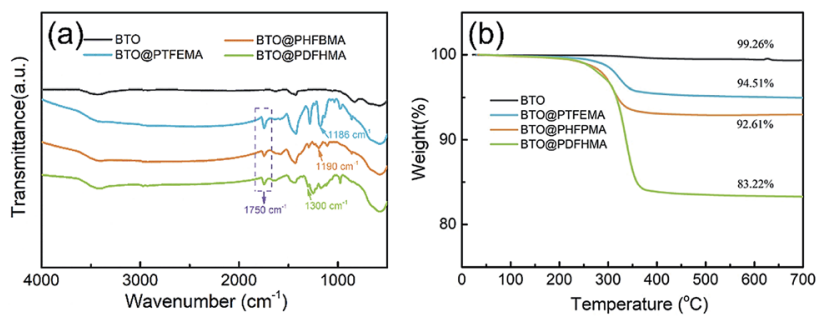

Fig. 2 (a) FTIR spectra and (b) TGA curves of the BTOafluoronanoparticles. methacrylate polymer have been successfully coated onto the surface of BTO_nps.

To further characterize the shell polymer structure of nanoparticles, TGA experiments have been conducted, as shown in Fig. 2b. With temperature elevated to $700{ }^{\circ} \mathrm{C}$, pristine BTO_nps barely shows weight loss. In contrast, weights of all three types of core-shell structured BTO_nps begin to decrease above $200{ }^{\circ} \mathrm{C}$, and then sharply drop after $300{ }^{\circ} \mathrm{C}$, which results from the thermal decomposition of fluoro-polymer. With complete thermal decomposition, the weight loss at $700{ }^{\circ} \mathrm{C}$ of the nanoparticles are about $0.74 \%, 5.49 \%, 7.39 \%$, and $16.78 \%$ for pristine BTO, BTO@PTFEMA, BTO@PHFBMA, and BTO@PDFHMA, respectively. The results also show that there is a certain amount of fluoro-polymer shell around the BTO_nps, which is consistent with the TEM results.

The BTO/P(VDF-HFP) and BTO@fluoro-polymer/P(VDF-HFP) polymer nanocomposites, where the volume fraction of nanofillers is fixed at $15 \mathrm{wt} \%$, are prepared by the nonequilibrium processing method. ${ }^{34}$ Firstly, the BTO/P(VDF-HFP) and BTO@fluoro-polymer/P(VDF-HFP) composite nanofibers are prepared by a modified electrospinning process. As shown in Fig. 3, the composite nanofibers are smooth, beads-free and of high uniformity. However, some agglomerates of BTO_nps are readily distinguished in the $\mathrm{BTO} / \mathrm{P}$ (VDF-HFP) composite nanofibers (Fig. 3a), which is attributed to the poor compatibility between inorganic particles and organic polymers. In stark contrast, more homogeneous dispersion of BTO@fluoropolymer within the P(VDF-HFP) nanofibers could be observed in Fig. 3b-d. But, there still are a slight amount of BTO_nps agglomerates in BTO@PTFEMA/P(VDF-HF) nanofibers. With increasing length of fluoro-polymer side chain, more segment interactions occur between the nanoparticle and the matrix, make the distribution of the BTO@fluoro-polymer nanoparticles more uniform in the $\mathrm{P}$ (VDF-HFP) nanofibers. In these four kinds of BTO_nps, BTO@PDFHMA exhibits the best dispersion state. These results indicate that the dispersion of nanoparticles could be greatly improved through introducing a polymer transition layer between BTO_nps and P(VDF-HFP) matrix, verifying the feasibility of core-shell strategies.

After hot-pressing treatment near the melting temperature of $\mathrm{P}$ (VDF-HFP), the BTO/P(VDF-HFP) and BTO@fluoro-polymer/ $\mathrm{P}(\mathrm{VDF}-\mathrm{HFP})$ fibrous mats are transformed into dense nanocomposite films, and the thickness of the films is around $12 \mu \mathrm{m}$

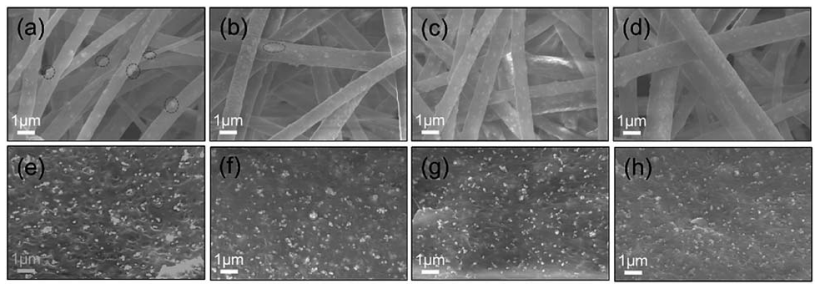

Fig. 3 SEM images of BTO@fluoro-polymer/P(VDF-HFP) nanocomposite fibers $(a-d)$ and cross sections (e-h) of BTOafluoropolymer/P(VDF-HFP) nanocomposite films with different BTOQfluoro-polymer nanoparticles: BTO (a and e), BTO (APTFEMA (b and f), BTO@PHFBMA (c and g), BTO@PDFHMA ( $d$ and $h$ ). 
(Fig. S1 $\dagger$ ). As evidenced by the cross-sectional SEM images of BTO/P(VDF-HFP) films (Fig. 3e), the as-received BTO_nps embedded in polymer matrix show severe agglomeration and are surrounded by the air instead of the polymer matrix. Comparatively, for composites containing BTO@fluoropolymer particles, there is no finding of particle-matrix debonding and nanoparticles dispersion degree is also improved (Fig. 3f-h). As the fluoro-polymers with branched chain share similar structure with $\mathrm{P}(\mathrm{VDF}-\mathrm{HFP})$, it can provide strong interface adhesion to the particles by interaction with the $\mathrm{P}($ VDF-HFP) segment, and the adverse effects caused by poor interface are also weakened. Therefore, the compatibility between particles and matrix are enhanced, and agglomerations are overcome in composites containing BTO@fluoropolymer particles. For instance, PDFHMA has the longest branched chain among three fluoroalkyl methacrylate polymers, which means more chains involved in and stronger interaction between BTO@PDFHMA and P(VDF-HFP). As a result, the best uniform dispersion is achieved in BTO@PDFHMA/P(VDF-HFP).

The frequency dependence of room-temperature dielectric permittivity and dielectric loss for P(VDF-HFP)-based nanocomposite films is presented in Fig. 4a. It can be seen from Fig. 4a that the dielectric constants of the P(VDF-HFP)-based nanocomposites with 15 wt\% of BTO_nps or BTO@fluoropolymer are improved over the whole frequency range from $10^{2} \mathrm{~Hz}$ to $10^{7} \mathrm{~Hz}$ compared with pure $\mathrm{P}(\mathrm{VDF}-\mathrm{HFP})$. Fig. $4 \mathrm{~b}$ shows the comparison of $\varepsilon_{\mathrm{r}}$ for different P(VDF-HFP)-based nanocomposite and pure $\mathrm{P}(\mathrm{VDF}-\mathrm{HFP})$ at the same frequency. For instance, at a fixed frequency of $10^{3} \mathrm{~Hz}$, the $\varepsilon_{\mathrm{r}}$ of the pure $\mathrm{P}(\mathrm{VDF}-$ HFP) is only 10.9 , while the $\varepsilon_{\mathrm{r}}$ of the BTO/P(VDF-HFP), BTO@PTFEMA/P(VDF-HFP), BTO@PHFBMA/P(VDF-HFP), and BTO@PDFHMA/P(VDF-HFP) nanocomposites is 11.7, 12, 12.4 and 13, respectively. At other fixed frequencies, the $\varepsilon_{\mathrm{r}}$ of the nanocomposites is higher than that of pure $\mathrm{P}(\mathrm{VDF}-\mathrm{HFP})$. It is obvious that the increase in dielectric constant of $\mathrm{BTO} / \mathrm{P}(\mathrm{VDF}-$ HFP) nanocomposites could be attributed to the high dielectric constant BTO_nps. In addition, Maxwell-Wagner-Sillars
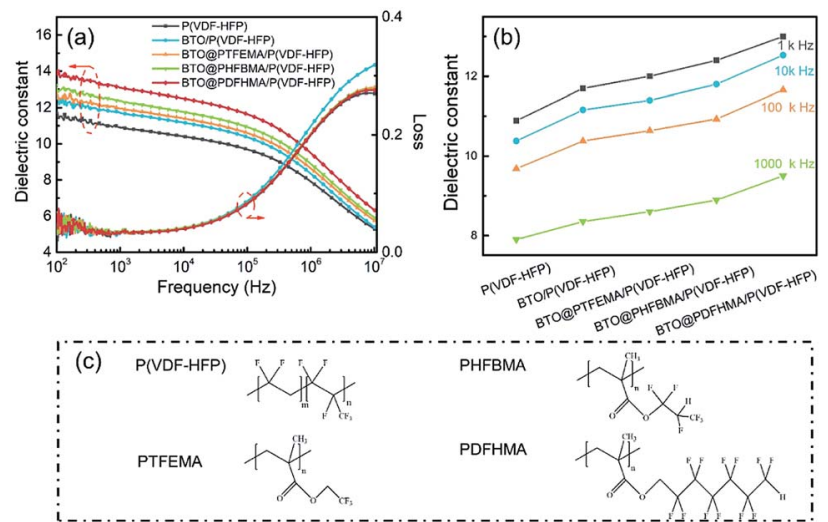

Fig. 4 (a) Frequency dependence of dielectric constant and dielectric loss and (b) comparison of dielectric constants at a fixed frequency for P(VDF-HFP)-based nanocomposite films. (c) The fluoro-polymer shells and P(VDF-HFP) segment chain structure.
(MWS) interfacial polarization is ubiquitous for polymer nanodielectrics, which increases the overall polarization by enhancing dielectric constant. ${ }^{38,39}$ For BTO@fluoro-polymer/ $\mathrm{P}(\mathrm{VDF}-\mathrm{HFP})$ nanocomposites, by introducing a shell structure between BTO and polymer, the enhancement of interfacial compatibility leads to a homogeneous nanoparticle dispersion and more compact interfaces. Therefore, more interfaces provide higher interfacial polarization, and result in dielectric performance improvement of the BTO@fluoro-polymer/P(VDFHFP) nanocomposites.

Fig. 4a also shows the dielectric loss tangent of P(VDF-HFP)based nanocomposite films with different types of BTO_nps. As seen, the BTO@fluoro-polymer/P(VDF-HFP) nanocomposites exhibit a relatively low dielectric loss comparable with pristine $\mathrm{P}(\mathrm{VDF}-\mathrm{HFP})$. While, the dielectric loss of $\mathrm{BTO} / \mathrm{P}(\mathrm{VDF}-\mathrm{HFP})$ nanocomposite is higher than that of the pristine $\mathrm{P}(\mathrm{VDF}-$ HFP), especially at high frequency. As we known, the dielectric loss of $\mathrm{P}(\mathrm{VDF}-\mathrm{HFP})$-based polymer at high frequency is ascribed to the segmental motions within the amorphous phase of PVDF. ${ }^{40}$ Compared to BTO@fluoro-polymer/P(VDF-HFP), poor bonding between BTO_nps and polymer matrix in BTO/ $\mathrm{P}$ (VDF-HFP) nanocomposites allows the polymer segments at the interface to act more freely, which leads to a higher dielectric loss.

Tight bonding between nanofillers and polymer matrices in the BTO@fluoro-polymer/P(VDF-HFP) not only helps to decrease their low-field dielectric loss, but is also beneficial to suppress their high-field electric conduction. Leakage current of all the composite films as a function of electric field are plotted in Fig. 5a. As seen, the BTO/P(VDF-HFP) nanocomposite exhibits the highest leakage current over the whole electric field range, ascribed to the increased amount of charge carriers brought by structural defects (e.g. agglomerations and air holes). For BTO@fluoro-polymer/P(VDF-HFP) composites, the leakage current is significantly suppressed, which benefits from the decreased charge carriers density due to the more homogeneous dispersion and the restricted movement of charge carriers by transition layer. What's more, better suppression of leakage current is achieved due to the fact that longer side chains increase the range of interaction between the transition layer and the P(VDF-HFP) matrix. Hence, BTO@PDFHMA/ $\mathrm{P}(\mathrm{VDF}-\mathrm{HFP})$ exhibits the lowest leakage current, which is comparable with or even lower than that of pristine $\mathrm{P}(\mathrm{VDF}-$ HFP).
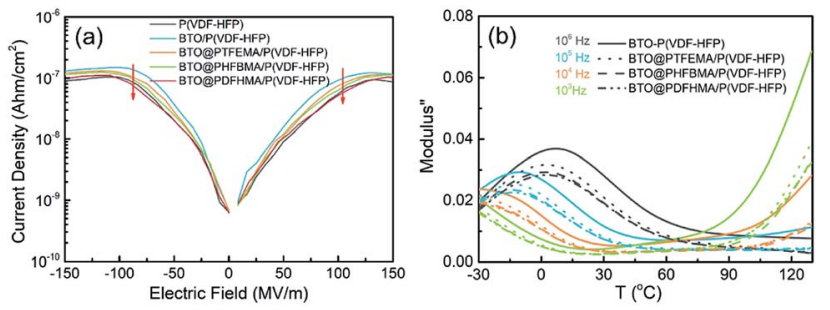

Fig. 5 (a) The variation of leakage current on electric fields and (b) the temperature-dependent and frequency-dependent imaginary electric modulus spectra for P(VDF-HFP)-based nanocomposite films. 
To further investigate the effect of the fluorine-containing shell branches on the motion behavior of P(VDF-HFP) segments, the temperature dependent and frequency dependent of the $M^{\prime \prime}$ is provided in Fig. 5b. Obviously, it can be observed from Fig. 5b that there are two relaxation peaks. The relaxation peak in low temperature region on the left corresponds to the P(VDF-HFP) segmental motions in amorphous regions, and as the temperature increases, the segment motion becomes easier, which makes the relaxation peak shift to high frequency. ${ }^{\mathbf{4 1 , 4 2}}$ More importantly, compared with BTO/P(VDFHFP) nanocomposites, the relaxation peak intensity of BTO@fluoro-polymer/P(VDF-HFP) has significantly decreased, especially for BTO@PDFHMA/P(VDF-HFP), the lowest among the four samples. The main reason is that the segmental motion of $\mathrm{P}$ (VDF-HFP) is restricted by the fluoro-polymer shells, and the longer the fluoro-polymer branches, the more obvious the restrict effect. In addition to restrict the segmental motion of $\mathrm{P}$ (VDF-HFP), fluoro-polymer branches also act as deep traps to capture free charge carriers to improve the insulation performance of nanocomposites. As can be seen from Fig. S2, $\dagger$ the conductivity of BTO@fluoro-polymer/P(VDF-HFP) nanocomposites have been reduced by one order of magnitude compared to the BTO/P(VDF-HFP) nanocomposites, which indicates that the fluoroalkyl methacrylate polymer shell can suppress the electrical conductivity, and this is also consistent with the previous results.

In order to explore the effects of the fluorine-containing shell branches on the motion of $\mathrm{P}(\mathrm{VDF}-\mathrm{HFP})$ segments, first-principle calculations is performed and the results are presented in Fig. 6 . As seen, the charge transfer between different fluoroalkyl methacrylate monomers and the (001) surface of BTO are well distinguished. For TFEMA, HFBMA, DFHMA, obvious charge accumulation regions and charge depletion regions can be found around $\mathrm{C}=\mathrm{O}$ after interaction with the BTO_nps, and this charge transfer region also responds to the internal atoms of BTO_nps. It is worth noting that for the DFHMA with the longest fluorine-containing segment, in addition to its obvious charge transfer between the BTO_nps, the long fluorinecontaining segment will extend outward, providing a point of action for the connection of the particles to the matrix. Therefore, methyl methacrylate with long fluorine-containing segment can not only make a strong interaction with BTO, but also make use of the interaction between its fluorinecontaining branch chain and matrix chain segment to make the particles more closely connected with the matrix.

In addition to the dielectric constant and dielectric loss mentioned above, another important parameter affecting the energy storage density of nanocomposite materials is the breakdown strength. In this study, the $E_{\mathrm{b}}$ of the pure P(VDFHFP) and nanocomposites are measured using a twoparameter Weibull distribution functions analysis. The Weibull distribution function is described as: $P(E)=1-\exp [-(E)$ $\left.\left.E_{\mathrm{b}}\right)^{\beta}\right]$, where $E$ is experimental breakdown strength; $E_{\mathrm{b}}$ is calculated from the Weibull distribution that refers to the breakdown strength at the cumulative failure probability of $63.2 \% ; \beta$ is a shape parameter to assess the degree of data dispersion and is also a standard for the quality of dielectric

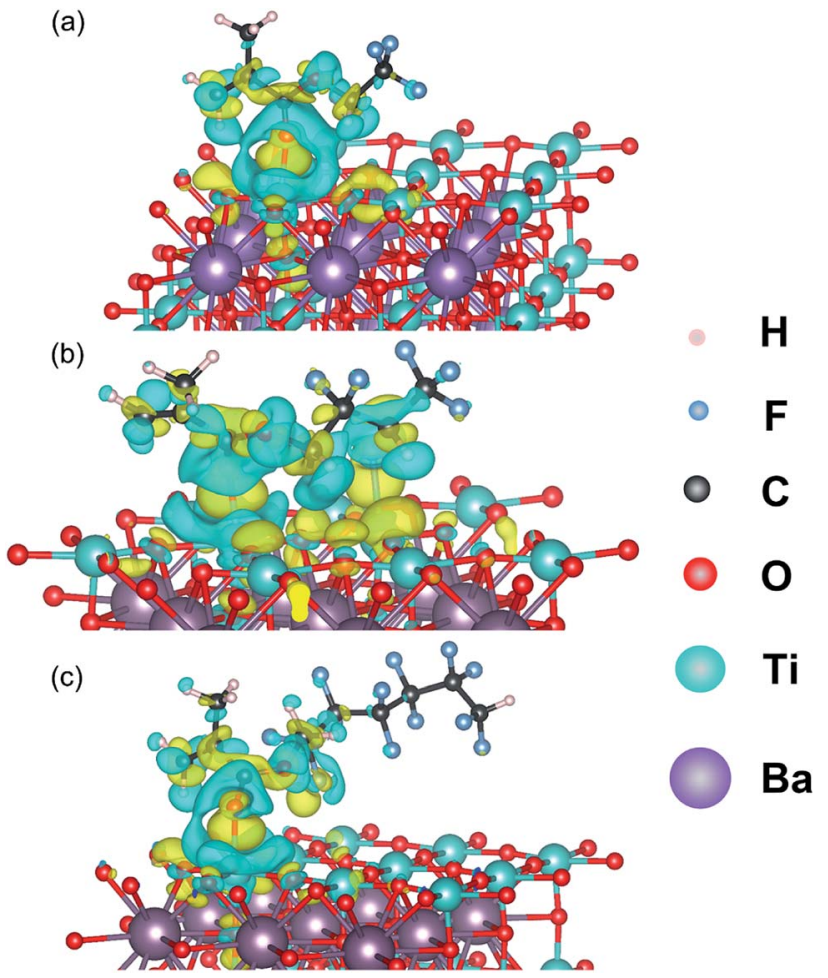

Fig. 6 Computed charge density difference of (a) TFEMA, (b) HFBMA, (c) DFHMA absorbed BTO-001. The yellow and blue isosurfaces represent charge accumulation and depletion in the space, respectively.

performance. ${ }^{43}$ The Weibull statistical analysis results of the nanocomposite films are shown in Fig. 7. As we can see, the $E_{\mathrm{b}}$ of $\mathrm{BTO} / \mathrm{P}(\mathrm{VDF}-\mathrm{HFP})$ is only $492 \mathrm{MV} \mathrm{m}^{-1}$. The difference in dielectric properties between the filler and the matrix causes the electric field to be concentrated at the interface between them, while the interface between the unmodified particles and the polymer matrix is weakly bonded, leading to the low $E_{\mathrm{b}}$ of BTO/ $\mathrm{P}(\mathrm{VDF}-\mathrm{HFP})$. However, the $E_{\mathrm{b}}$ of the other three nanocomposites

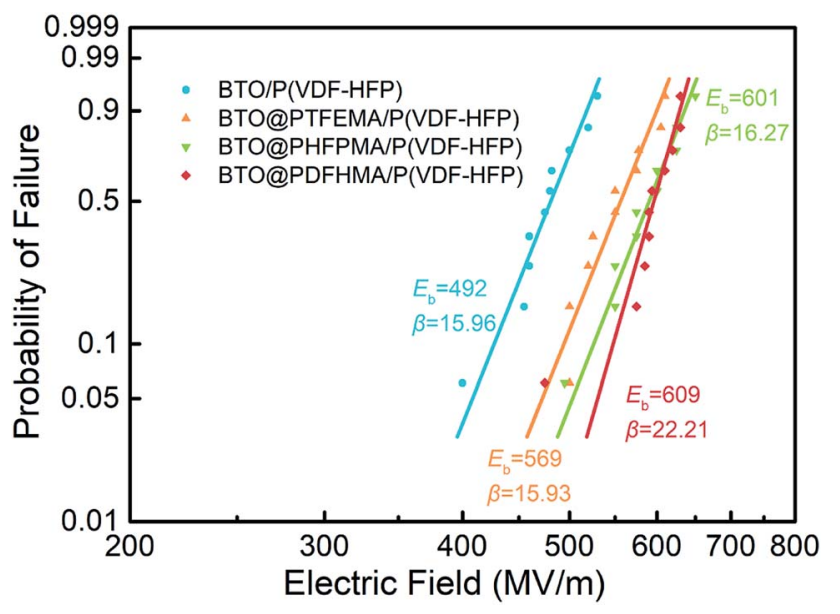

Fig. 7 Weibull distributions of electric breakdown strength at room temperature for P(VDF-HFP)-based nanocomposite films. 
containing BTO@fluoro-polymer has a substantial enhancement. For instance, the $E_{\mathrm{b}}$ for BTO@PTFEMA/P(VDF-HFP), BTO@PHFBMA/P(VDF-HFP), and BTO@PDFHMA/P(VDF-HFP) is $569 \mathrm{MV} \mathrm{m}^{-1}(\beta \sim 15.9), 601 \mathrm{MV} \mathrm{m}^{-1}(\beta \sim 16.3)$ and $609 \mathrm{MV}$ $\mathrm{m}^{-1}(\beta \sim 22.2)$, respectively. The enhancement in breakdown strength can be explained as the alleviation of distorted electric field near the particle/matrix interfaces. By introducing a fluoroalkyl methacrylate polymer transition layer between BTO_nps and P(VDF-HFP) matrix, the BTO_nps are more closely connected to the P(VDF-HFP) matrix, and the mismatch between particles and matrix caused by the differences in dielectric properties will be eliminated. Therefore, the $E_{\mathrm{b}}$ of these three nanocomposites are greatly improved, and longer fluorinated branched chains can significantly increase the $E_{\mathrm{b}}$. The results not only demonstrate that the introduction of fluoro-polymer shells has a positive effect on the breakdown strength of the nanocomposites films, but also indicate that longer shell molecular chain branches have positive impact on the breakdown strength of the nanocomposites.

The energy densities and efficiencies of these nanocomposites are calculated from the electric displacement-electric field $(D-E)$ loops (see $D-E$ loops and calculation details in Fig. S3 $\dagger$ ), and the discharged energy densities are plotted as a function of electric field in Fig. 8a. Owing to concurrently enhanced $\varepsilon_{\mathrm{r}}$ and $E_{\mathrm{b}}$ that induced by core-shell particle structure in the BTO@fluoro-polymer/P(VDF-HFP) nanocomposites, the $U_{\text {e }}$ are remarkably increased compared with the BTO/P(VDFHFP) nanocomposite. For instance, a highest $U_{\mathrm{e}} \sim 16.8 \mathrm{~J}$ $\mathrm{cm}^{-3}$ at $609 \mathrm{MV} \mathrm{m}^{-1}$ is obtained in BTO@PDFHMA/P(VDF-HFP) nanocomposite, which is 1.46 times $U_{\mathrm{e}}$ of $\mathrm{BTO} / \mathrm{P}(\mathrm{VDF}-\mathrm{HFP})$ nanocomposite $\left(U_{\mathrm{e}} \sim 11.5 \mathrm{~J} \mathrm{~cm}^{-3}\right.$ at $\left.492 \mathrm{MV} \mathrm{m}^{-1}\right)$. The $U_{\mathrm{e}}$ of BTO@PTFHMA/P(VDF-HFP) $\left(\sim 12.5 \mathrm{~J} \mathrm{~cm}^{-3}\right.$ at $\left.569 \mathrm{MV} \mathrm{m}^{-1}\right)$ and BTO@PHFBMA/P(VDF-HFP) $\left(\sim 15.1 \mathrm{~J} \mathrm{~cm}^{-3}\right.$ at $\left.601 \mathrm{MV} \mathrm{m}^{-1}\right)$ are also higher than that of $\mathrm{BTO} / \mathrm{P}(\mathrm{VDF}-\mathrm{HFP})$ nanocomposite. Moreover, $\eta$ is also an important parameter for evaluating the dielectric performances, as a high $\eta$ is necessary when dielectric materials are applied to electrical insulation and energy storage devices. Usually, for P(VDF-HFP) based nanocomposite, it has a low discharge efficiency $(\eta)$ of $\sim 60 \%$, which means that only about $60 \%$ of the energy can be used during energy storage and release. The massive wasted energy will be converted into surface energy of breakdown paths and Joule heat, leading to crippled breakdown strength and lifetime. The variation curves of $\eta$ versus electric field for nanocomposites are also present in
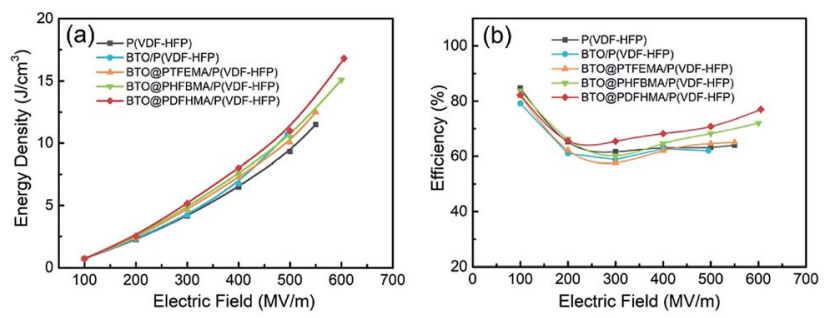

Fig. 8 (a) Discharged energy density and (b) charge-discharge efficiency of the nanocomposite films as a function of the electric field calculated from $D-E$ loops in Fig. S3, $\uparrow$ respectively.
Fig. 8b. Notably, the BTO/P(VDF-HFP) nanocomposite has the lowest $\eta$ of $\sim 62 \%$ among these four types of P(VDF-HFP) based nanocomposites, which is due to the high dielectric loss during charge/discharge cycling caused by defects that introduced with BTO_nps, such as voids, ions and impurities. Compared with pristine BTO_nps, the BTO@fluoroalkyl methacrylate coreshell structured nanoparticles can restrict the movement of free carriers to suppress the conductivity loss, and also reduce the generation of voids by making use of their similarity to the matrix. Therefore, higher discharge efficiencies have been achieved in nanocomposites with BTO@fluoro-polymer particles, especially for BTO@PDFHMA/P(VDF-HFP) nanocomposite exhibiting a rather high $\eta \sim 78 \%$.

\section{Conclusion}

We have selected three kinds of fluoro-polymers as the transition layer between the particles and the polymer matrix, and use a simple and direct method to coat the transition layer on the surface of the particles to form a stable and dense core-shell structure. The selected fluoro-polymer all have fluorinecontaining branched chains, and the side chain length of fluoro-polymer on the properties of polymer nanocomposites are investigated. The side chains of fluoro-polymer have similar segment structure to P(VDF-HFP), which can interact well with the $\mathrm{P}(\mathrm{VDF}-\mathrm{HFP})$ molecular chain and improve the compatibility between the BTO_nps and P(VDF-HFP). Benefiting from this, the dispersions of BTO_nps are well improved, and good interaction between the segments can make BTO_nps embedded into matrix more tightly. In addition, the core-shell structure can reduce the number of free carriers to suppress the leakage current and enhance the breakdown strength. The longer side chain lengths of fluoro-polymer also have a more positive impact on the performance of the nanocomposites. As a result, with the improvement of these properties, the efficiencies and energy densities of the nanocomposites containing the BTO@fluoro-polymer nanoparticles are found to be significantly increased. These results show that the core-shell structure of fluoro-polymer can combine both the advantages of inorganic particles and polymer matrix, and improve the dielectric properties of nanocomposites. This work also provides a novel and simple path for the preparation of coreshell nanocomposites toward high energy storage density.

\section{Conflicts of interest}

There are no conflicts to declare.

\section{Acknowledgements}

This work was supported by the NSF of China (Grant No. 51625202, and 51572141).

\section{References}

1 B. Chu, X. Zhou, K. Ren, B. Neese, M. Lin, Q. Wang, F. Bauer and Q. M. Zhang, Science, 2006, 313, 334-336. 
2 X. Zhang, Y. Shen, B. Xu, Q. Zhang, L. Gu, J. Jiang, J. Ma, Y. Lin and C.-W. Nan, Adv. Mater., 2016, 28, 2055-2061.

3 Q. Li, L. Chen, M. R. Gadinski, S. Zhang, G. Zhang, H. Li, A. Haque, L.-Q. Chen, T. N. Jackson and Q. Wang, Nature, 2015, 523, 576-580.

4 Z.-M. Dang, J.-K. Yuan, S.-H. Yao and R.-J. Liao, Adv. Mater., 2013, 25, 6334-6365.

5 Q. Chen, Y. Shen, S. Zhang and Q. M. Zhang, Annu. Rev. Mater. Res., 2015, 45, 433-458.

6 M. Marz, A. Schletz, B. Eckardt, S. Egelkraut and H. Rauh, CIPS, 2010, pp. 1-10.

7 T. R. Jow, F. W. MacDougall, J. B. Ennis, X. H. Yang, M. A. Schneider, C. J. Scozzie, J. D. White, J. R. MacDonald, M. C. Schalnat, R. A. Cooper and S. P. S. Yen, $P P C, 2015$, pp. 1-7.

8 Z. Pan, L. Yao, J. Zhai, X. Yao and H. Chen, Adv. Mater., 2018, 30, 1705662.

9 Y. Shen, Y. Hu, W. Chen, J. Wang, Y. Guan, J. Du, X. Zhang, J. Ma, M. Li, Y. Lin, L.-q. Chen and C.-W. Nan, Nano Energy, 2015, 18, 176-186.

10 Y. Wang, L. Wang, Q. Yuan, J. Chen, Y. Niu, X. Xu, Y. Cheng, B. Yao, Q. Wang and H. Wang, Nano Energy, 2018, 44, 364370.

11 M. Rabuffi and G. Picci, IEEE Trans. Plasma Sci., 2002, 30, 1939-1942.

12 V. Tomer, E. Manias and C. A. Randall, J. Appl. Phys., 2011, 110.

13 V. K. Thakur, M.-F. Lin, E. J. Tan and P. S. Lee, J. Mater. Chem., 2012, 22, 5951-5959.

14 H. Luo, D. Zhang, C. Jiang, X. Yuan, C. Chen and K. Zhou, ACS Appl. Mater. Interfaces, 2015, 7, 8061-8069.

15 Y. Song, Y. Shen, H. Liu, Y. Lin, M. Li and C.-W. Nan, J. Mater. Chem., 2012, 22, 16491-16498.

16 Q. Wang and L. Zhu, J. Polym. Sci., Part B: Polym. Phys., 2011, 49, 1421-1429.

17 J. Y. Li, L. Zhang and S. Ducharme, Appl. Phys. Lett., 2007, 90, 132901.

18 H. Luo, X. Zhou, C. Ellingford, Y. Zhang, S. Chen, K. Zhou, D. Zhang, C. R. Bowen and C. Wan, Chem. Soc. Rev., 2019, 48, 4424-4465.

19 Y. Niu, Y. Bai, K. Yu, Y. Wang, F. Xiang and H. Wang, ACS Appl. Mater. Interfaces, 2015, 7, 24168-24176.

20 R. Zhao, J. Zhao, L. Wang and Z.-M. Dang, J. Appl. Polym. Sci., 2015, 132.

21 P. Kim, N. M. Doss, J. P. Tillotson, P. J. Hotchkiss, M.-J. Pan, S. R. Marder, J. Li, J. P. Calame and J. W. Perry, ACS Nano, 2009, 3, 2581-2592.
22 P. Kim, S. C. Jones, P. J. Hotchkiss, J. N. Haddock, B. Kippelen, S. R. Marder and J. W. Perry, Adv. Mater., 2007, 19, 1001-1005.

23 K. Yu, H. Wang, Y. Zhou, Y. Bai and Y. Niu, J. Appl. Phys., 2013, 113.

24 Q. Li, G. Zhang, F. Liu, K. Han, M. R. Gadinski, C. Xiong and Q. Wang, Energy Environ. Sci., 2015, 8, 922-931.

25 Y. Niu, F. Xiang, Y. Wang, J. Chen and H. Wang, Phys. Chem. Chem. Phys., 2018, 20, 6598-6605.

26 S. Siddabattuni, T. P. Schuman and F. Dogan, ACS Appl. Mater. Interfaces, 2013, 5, 1917-1927.

27 L. Xie, X. Huang, C. Wu and P. Jiang, J. Mater. Chem., 2011, 21, 5897-5906.

28 M. N. Tchoul, S. P. Fillery, H. Koerner, L. F. Drummy, F. T. Oyerokun, P. A. Mirau, M. F. Durstock and R. A. Vaia, Chem. Mater., 2010, 22, 1749-1759.

29 X. Pang, Y. He, B. Jiang, J. Iocozzia, L. Zhao, H. Guo, J. Liu, M. Akinc, N. Bowler, X. Tan and Z. Lin, Nanoscale, 2013, 5, 8695-8702.

30 K. Jiang, C. Ye, P. Zhang, X. Wang and Y. Zhao, Macromolecules, 2012, 45, 1346-1355.

31 K. Yang, X. Huang, L. Xie, C. Wu, P. Jiang and T. Tanaka, Macromol. Rapid Commun., 2012, 33, 1921-1926.

32 L. Xie, X. Huang, Y. Huang, K. Yang and P. Jiang, J. Phys. Chem. C, 2013, 117, 22525-22537.

33 H. Luo, S. Chen, L. Liu, X. Zhou, C. Ma, W. Liu and D. Zhang, ACS Sustainable Chem. Eng., 2018, 7, 3145-3153.

34 J. Jiang, X. Zhang, Z. Dan, J. Ma, Y. Lin, M. Li, C.-W. Nan and Y. Shen, ACS Appl. Mater. Interfaces, 2017, 9, 29717-29731.

35 S. K. Papadopoulou, C. Tsioptsias, A. Pavlou, K. Kaderides, S. Sotiriou and C. Panayiotou, Colloids Surf., A, 2011, 387, 71-78.

36 B. Jiang, L. Zhang, J. Shi, S. Zhou, B. Liao, H. Liu, J. Zhen and H. Pang, J. Fluorine Chem., 2013, 153, 74-81.

37 J. Tan, W. Liu and Z. Wang, Prog. Org. Coat., 2017, 105, 353361.

38 R. Tamura, E. Lim, T. Manaka and M. Iwamoto, J. Appl. Phys., 2006, 100, 114515.

39 Z.-M. Dang, L. Wang, Y. Yin, Q. Zhang and Q.-Q. Lei, Adv. Mater., 2007, 19, 852-857.

40 P. Fruebing, F. Wang and M. Wagener, Appl. Phys. A: Mater. Sci. Process., 2012, 107, 603-611.

41 L. Xie, X. Huang, B.-W. Li, C. Zhi, T. Tanaka and P. Jiang, Phys. Chem. Chem. Phys., 2013, 15, 17560-17569.

42 L. Yu and P. Cebe, J. Polym. Sci., Part B: Polym. Phys., 2009, 47, 2520-2532.

43 P. Khanchaitit, K. Han, M. R. Gadinski, Q. Li and Q. Wang, Nat. Commun., 2013, 4, 2845. 